\title{
Year in review 2012: Critical Care - cardiology
}

\author{
Daniel De Backer
}

Abstract
In this review I discuss key research papers in
cardiology and intensive care published in Critical Care
during 2012 with related studies published in other
journals quoted whenever appropriate. These studies
are grouped into the following categories:
cardiovascular therapies, mechanical therapies,
pathophysiologic mechanisms, hemodynamic
monitoring, ultrasound in respiratory failure,
microcirculation, and miscellaneous.

\section{Review}

\section{Cardiovascular therapies}

The impact of extracellular acidosis on myocardial performance and responsiveness to inotropic stimulation is still the topic of intense debate. While mild acidosis seems not to be associated with myocardial depression or hyporesponsiveness in isolated hearts obtained from healthy animals, failing hearts may be more sensitive to acidosis. Isolated isometrically twitching human myocardial trabeculae recovered from end-stage failing hearts were exposed to $\mathrm{pH} 7.40$ and 7.20 [1]. This mild metabolic acidosis caused a 26\% depression in twitch-force amplitude without impairment of relaxation. During isoproterenol stimulation, the force amplitude was comparable at $\mathrm{pH}$ levels but the dose response curve was shifted to the right. These results imply that higher doses of adrenergic agents are required to reach the same cardiac output in the presence of mild acidosis. These findings should be confirmed by in vivo studies.

The type of intravenous fluid is also a topic of intense debate. In a pig model of severe normovolemic hemodilution, the effects of tetrastarch, gelatin, hetastarch and Ringer's solution on critical hemoglobin were evaluated [2]. Hemodilution could reach lower critical hemoglobin levels with tetrastarch $(2.1 \pm 0.4 \mathrm{~g} / \mathrm{dL})$ and gelatins $(2.7 \pm 0.6 \mathrm{~g} / \mathrm{dL})$ than with hetastarch $(3.0 \pm 0.6 \mathrm{~g} / \mathrm{dL})$ and Ringer's solution

Correspondence: ddebacke@ulb.ac.be

Department of Intensive Care, Erasme University Hospital, Université Libre de Bruxelles, B-1070, Brussels, Belgium
$(3.7 \pm 0.6 \mathrm{~g} / \mathrm{dL})$. The fluids differed in their volume effects, viscosity, and extravasation rate and these factors may have influenced microcirculatory perfusion and hence critical hemoglobin.

\section{Mechanical therapies}

A very important trial challenged the use of intra-aortic balloon pump (IABP) counterpulsation [3]. In this randomized trial including 600 patients with cardiogenic shock complicating acute myocardial infarction, IABP failed to improve mortality at 30 days (40\% versus $41 \%$, $P=0.69$ ). There were no significant differences in the time to hemodynamic stabilization, including blood lactate levels, and this was not affected by the dose and duration of catecholamine therapy. Surprisingly, the rate of complications did not differ either, including bleeding and ischemic events. It should be highlighted that this trial included patients for whom an early revascularization strategy was planned, which may explain, at least in part, the relatively low mortality in both groups. These results may not apply in other types of cardiogenic shock.

A case control study evaluated the potential role of descending aortic calcifications on complications after use of IABP in cardiac surgery [4]. In a series of 459 patients after cardiac surgery, perioperative IABP and descending aortic atheroma were independently associated with perioperative kidney injury and in-hospital mortality.

Use of IABP is sometimes proposed as an adjunct to extracorporeal membrane oxygenation (ECMO) in order to preserve myocardial and cerebral perfusion. In an experimental model of prolonged cardiac arrest, the effects of IABP as well as ECMO cannulation site on cerebral and coronary perfusion were investigated [5]. Both femoro-femoral and femoro-subclavian ECMO provided adequate brain perfusion and oxygenation but femorofemoral ECMO offered better coronary perfusion than femoro-subclavian ECMO. Addition of IABP to ECMO worsened coronary perfusion, especially in femorofemoral ECMO. These experimental data do not support the adjunction of IABP to ECMO in cardiac arrest. 


\section{Pathophysiologic mechanisms}

The measurement of blood lactate levels is commonly used to guide resuscitation in shock but lactate can be of variable origin. Early after admission, lactate is predominantly of anaerobic origin but rapidly other causes of hyperlactatemia may play a role. At the onset of shock, $69 \%$ of patients with cardiogenic shock and $76 \%$ of patients with septic shock presenting hyperlactatemia also had a high lactate to pyruvate ratio [6]. The time course of the lactate/pyruvate ratio decreased more slowly in non-survivors and in patients with cardiogenic shock than in the others. When lactate is not of anaerobic origin, it can arise either from aerobic causes, mostly accelerated glycolysis, or a decrease in lactate clearance. Lactate metabolism was investigated using muscle microdialysis, exogenous lactate infusion and stable isotopes in a series of healthy volunteers challenged with low dose endotoxin [7]. Plasma lactate concentration rapidly but modestly increased, never exceeding $2 \mathrm{mmol} / \mathrm{l}$, in relation to the increase in resting energy expenditure. Muscle lactate production and total body lactate clearance remained constant. It is likely that immune cells may be an important source of lactate in sepsis. This is in agreement with previous data showing increased lactate production in white blood cells in sepsis [8].

Metformin can also lead to severe lactic acidosis associated with organ dysfunction. Metformin intoxication is associated with a decrease in oxygen consumption (VO2), related to mitochondrial toxicity [9]. To evaluate the extent to which acidosis itself may explain the impaired mitochondrial function, lactic acid was infused in a pig model to achieve the same increase in lactate levels and decrease in $\mathrm{pH}$ as in metformin intoxicated animals [10]. Oxygen delivery was similar in the two groups but $\mathrm{VO} 2$ markedly decreased in metformin intoxication whereas it slightly increased with lactic acid infusion. Unfortunately, glucose levels also markedly differed between the two groups, and this may have impacted energy expenditure. Compared to sham animals, mitochondrial activity was markedly impaired in metformin intoxication. A similar alteration in mitochondrial activity was also observed in platelets obtained from humans with metforminrelated lactic acidosis [11]. These observations should not prevent physicians from using metformin, a drug that has been shown to have many useful effects when used appropriately. Of note, metformin administration may even attenuate ventilation-induced lung injury [12]. In a rabbit model of ventilation-induced lung injury, pre-treatment with metformin was associated with a reduction of lung edema and histologic lesions.

Carbon monoxide is an endogenous mediator with pleiotropic effects, including microvascular vasodilation, angiogenesis and cellular protection. Carbon monoxide production can be modulated in various directions in disease states. In a retrospective analysis of 868 patients admitted to a single ICU, carboxyhemoglobin levels were measured to evaluate endogenous carbon monoxide production [13]. Mean carboxyhemoglobin levels differed between admission categories, with the highest values observed in patients with renal and gastrointestinal disease and the lowest levels in neurological diseases. Patients who had higher severity scores on admission and non-survivors had lower minimal levels of carboxyhemoglobin during their stay in the ICU. These results indirectly suggest that endogenous carbon monoxide has a protective role.

\section{Hemodynamic monitoring}

\section{Cardiac output and volumetric measurements}

Transpulmonary thermodilution is frequently used to determine cardiac output and to evaluate extravascular lung water (EVLW) volume and intravascular volumes (global end diastolic volume (GEDV)). A new transpulmonary thermodilution system (VolumeView/EV1000 ${ }^{\text {ma }}$ system, Edwards Lifesciences, Irvine, CA, USA) was evaluated in 72 critically ill patients [14]. During 72 hours of observation, 443 sets of measurements were obtained with the new system, electronically recorded, converted into an analog resistance signal, and then re-analyzed by a PiCCO system (PiCCO2; Pulsion Medical Systems, Munich, Germany). For cardiac output, GEDV, and EVLW, the systems showed minimal bias $(0.2 \mathrm{~L} /$ minute, $29.4 \mathrm{ml}$ and $36.8 \mathrm{ml}$, respectively), and a low percentage error $(9.7 \%$, $11.5 \%$ and $12.2 \%$, respectively). Changes in these variables were adequately tracked. These systems thus seem to have equivalent performances. One may criticize the study design using re-analysis of signal obtained with one device by the alternative device, but it was ethically difficult to insert a second arterial line just for this purpose. Interest in measuring EVLW was highlighted in a trial including 51 patients with shock [15]. In addition to clinical evaluation, the measurement of EVLW by PiCCO increased the posttest odds ratio for the diagnosis of acute respiratory distress syndrome (ARDS) by 8 . EVLW was also higher in non-survivors. The impact of increases in EVLW on outcome was confirmed in a recent trial including $200 \mathrm{pa}-$ tients with ARDS [16].

Less invasive techniques of hemodynamic monitoring are gaining attractiveness. These are often validated in perioperative conditions but may sometimes be less reliable in more severe patients. Pulse wave amplitude was measured with a pneumatic cuff at finger extremity with a non-invasive arterial pulse contour device (Nexfin; BMeye, Amsterdam, The Netherlands) and compared to transpulmonary thermodilution (PiCCO) in a series of 45 patients with shock requiring administration of fluids [17]. Due to vasoconstriction, cardiac output could not be measured in seven patients. In the remaining 38 
patients, determination of arterial pressure and cardiac output was far from reliable due to large limits of agreement. Detection of changes in cardiac index was satisfactory when directional changes were considered (concordance of 76\%), although the magnitude of changes was not well tracked. The non-invasive arterial pulse contour device had a sensitivity of $43 \%$ (but good specificity) to detect changes in cardiac index in response to fluid challenge. These data question the reliability of this device in patients with shock.

\section{Evaluation of fluid responsiveness}

Respiratory variation in inferior vena cava diameter has been shown to predict fluid responsiveness in mechanically ventilated patients and it has been suggested that it may also be used in spontaneously breathing patients. In 40 spontaneously breathing patients, several echocardiographic indices were evaluated before and after fluid administration [18]. The predictive value of respiratory variations in inferior vena cava to predict fluid responsiveness was satisfactory with a receiver operating characteristics curve area of 0.77 , but this area did not differ from that of many static indices of preload. The best cutoff value for respiratory variation of inferior vena cava diameter was $40 \%$, which is higher than previously reported [19]. This threshold was quite specific for the response to fluids but lacked sensitivity, as 7 of the 20 responders to fluids had respiratory variations in inferior vena cava diameter of below $40 \%$. Reasons potentially explaining the lack of effectiveness of this index in spontaneously breathing patients were nicely reported in the accompanying editorial [19].

\section{Echocardiography}

Echocardiography is now recommended for the hemodynamic assessment of critically ill patients [20,21]. However, classical devices are usually large and heavy, and may take time to switch on, which may limit their use in some circumstances, and in particular in the emergency department. New pocket ultrasound devices have been developed, both for cardiac and non-cardiac ultrasound. In 151 patients admitted to the emergency department, Biais and colleagues [22] compared an ultra-miniaturized ultrasound device $\left(\operatorname{Vscan}^{\mathrm{Tu}}, \mathrm{GE}\right.$ Healthcare, Wauwatosa, WI, USA) and a conventional high-quality echocardiography system (Vivid $\mathrm{S}^{\mathrm{mm}}$, GE Healthcare) for focused cardiac ultrasonography. Image quality was sufficient with the pocket device to perform focused cardiac ultrasonography in all patients. There was a good agreement between both techniques for all items examined. Similar results were obtained in 94 critically ill patients [23]. In that trial left ventricular ejection fraction (LVEF) was independently assessed clinically by the attending physician and echocardiographically by two experienced intensivists trained in critical care echocardiography who used the pocket ultrasound and a fully featured device (CX50, Philips Healthcare, Eindhoven, The Netherlands). There was good concordance in LVEF estimation between the two echocardiographic devices, but clinical assessment of LVEF poorly correlated with echocardiographic assessment. Altogether, these data emphasize that clinical assessment of heart function is far from reliable and that pocket ultrasound devices can now be considered as a new stethoscope. Minimal training is needed; emergency physicians following a web-based learning module and 3 hours of proctored practical training showed a good concordance with an experienced cardiologist for focused echocardiographic examination [24].

Myocardial depression is frequently observed in sepsis and echocardiography is now the preferred way to identify it; several indices can be used for this. Longitudinal displacement of mitral annulus (Sa) was assessed by tissue Doppler in 60 patients with septic shock [25]. Compared to survivors, non-survivors exhibited significantly higher Sa. These data suggest either that myocardial hibernation occurs in sepsis as a protective phenomenon [26] or that non-survivors had higher myocardial stimulation (through higher endogenous and adrenergic stimulation) and lower afterload leading to an apparently better preserved myocardial function.

\section{Ultrasound in respiratory failure}

Echocardiography may have a place in identifying cardiac origins of weaning failure [27-29]. In addition, echocardiography may further help to discriminate between a systolic and diastolic component of heart dysfunction, which may lead to different therapies. Transthoracic echocardiography with Doppler tissue imaging was applied just before and 10 minutes after initiation of weaning attempts in 68 patients [30]. Twenty patients failed to be weaned from mechanical ventilation and a cardiac cause contributed to this in 15 of these. LVEF, as an index of systolic function, did not differ among weaning success and weaning failure. On the contrary, displacement of mitral annulus identified by tissue Doppler was lower in patients failing to be weaned, suggesting that diastolic impairment contributed markedly to weaning failure.

Lung ultrasonography is applied more and more for the evaluation of lung disease. It was applied in $98 \mathrm{pa}-$ tients presenting to the emergency department for flulike symptoms during the 2009 influenza A (H1N1) pandemic [31]. Lung ultrasonography had excellent diagnostic properties to detect lung infection (sensitivity 94\%, specificity $85 \%$, positive predictive value $87 \%$ and negative predictive value 93\%). More importantly, lung ultrasonography was abnormal in 16 patients with initially normal chest X-ray who later were proven to effectively 
have viral or bacterial pneumonia. Another group of investigators used lung ultrasonography to identify bacterial pneumonia in patients with H1N1 viral infection [32]. Viral pneumonia was associated with diffuse B line patterns only whereas bacterial pneumonia was characterized by the presence of consolidated areas with aeric bronchogram, associated or not with diffuse B line patterns. Admittedly this approach was somewhat cumbersome and required careful evaluation of anterior, lateral and posterior parts of the chest.

\section{Microcirculation}

The microcirculation is an area of continued interest. As alterations of microvascular perfusion in sepsis and their consequences are now recognized, new therapies are urgently needed. Enoxaparin acts not only as an anticoagulant but has marked anti-inflammatory properties. In rats, enoxaparin administration just after endotoxin administration, compared to placebo, better preserved endothelial function as reflected by a better preserved blood pressure and gut microvascular perfusion [33]. These results were further extended in a trial by another group of investigators demonstrating that enoxaparin decreased adhesion and rolling of white blood cells and, more importantly, decreased the severity of organ dysfunction in endotoxic rats [34].

Evaluation of endothelial function at the bedside can be done in several ways. Evaluation of brachial artery reactivity is one of these techniques. Flow and diameter of the vessels are evaluated with a high frequency vascular ultrasonic probe and vascular occlusion is performed with a cuff positioned on the same arm. Using this technique in 52 controls and 95 patients with severe sepsis, hyperemic velocity and flow-mediated dilation were found to be decreased in patients with sepsis [35]. Hyperemic velocity but not flow-mediated dilation was found to be associated with outcome. This test is simple and easy to reproduce at the bedside.

\section{Miscellaneous}

Acute kidney injury (AKI) is frequently observed after cardiac surgery, especially in patients with risk factors such as older age and diabetes. A preliminary trial suggested that bicarbonate administration could prevent post-operative AKI [36]. While confirmatory trials were on their way, some centers implemented this practice. In a before and after trial, administration of bicarbonate was not associated with reduction of AKI incidence [37]. If anything, bicarbonate administration was even associated with some detrimental effects, such as lower blood pressure and higher doses of vasopressor agents. These results were later confirmed by two randomized trials. In 92 patients with chronic kidney disease submitted to cardiac surgery, bicarbonate administration failed to prevent further deterioration of renal function [38]. A large scale multicenter randomized trial was stopped prematurely after inclusion of 350 patients for futility and potential harm [39]. More patients receiving bicarbonate developed AKI compared with control patients (48 versus $36 \%, P=0.032$ unadjusted, $P=0.120$ after adjustment). Hospital mortality was increased in patients receiving sodium bicarbonate compared to control (6.3 versus $1.7 \%, P=0.031)$. Altogether, these trials suggest that prophylactic use of sodium bicarbonate infusion to reduce the risk of AKI should be abandoned.

In patients with liver failure, the type of anticoagulation that should be applied during liver dialysis is still uncertain. Often liver dialysis is performed without anticoagulation but clotting of the system is frequent, decreasing its efficiency or even leading to paradoxical bleeding due to local consumption of the few available coagulation factors. Citrate anticoagulation is an attractive solution as it provides regional anticoagulation and does not have the bleeding risk of heparin. However, as citrate metabolism mostly occurs in liver and muscles there is a risk of citrate accumulation in these patients. Ten patients submitted to liver dialysis using the Molecular Adsorbents Recirculating System (MARS) were randomized in an open-label crossover trial of regional citrate anticoagulation versus anticoagulation-free liver dialysis [40]. None of the patients receiving citrate anticoagulation developed an event necessitating preterm cessation of MARS treatment. Four sessions without anticoagulation were interrupted preterm, one due to uncontrollable bleeding and three due to occlusive clotting of the extracorporeal blood circuit. There were no signs of citrate accumulation. Citrate anticoagulation may thus appear as a safe procedure for liver dialysis with MARS.

\section{Conclusion}

In conclusion, many studies published this year in the Journal have helped to increase our knowledge in these various fields.

\section{Abbreviations}

AKI: Acute kidney injury; ARDS: Acute respiratory distress syndrome; ECMO: Extracorporeal membrane oxygenation; EVLW: Extravascular lung water; GEDV: Global end diastolic volume; IABP: Intra-aortic balloon pump; LVEF: Left ventricular ejection fraction; MARS: Molecular Adsorbents Recirculating System; VO2: Oxygen consumption.

\section{Competing interests}

DDB has received materials for studies from Edwards Lifesciences, Pulsion, Cheetah Medical, BMeye, Vygon and Imacor in the last three years.

\section{Published: 22 Nov 2013}

\section{References}

1. Schotola H, Toischer K, Popov AF, Renner A, Schmitto JD, Gummert J, Quintel M, Bauer M, Maier LS, Sossalla S: Mild metabolic acidosis impairs the beta-adrenergic response in isolated human failing myocardium. Crit Care 2012, 16:R153. 
2. Pape A, Kutschker S, Kertscho H, Stein P, Horn O, Lossen M, Zwissler B, Habler O: The choice of the intravenous fluid influences the tolerance of acute normovolemic anemia in anesthetized domestic pigs. Crit Care 2012, 16:R69.

3. Thiele H, Zeymer U, Neumann FJ, Ferenc M, Olbrich HG, Hausleiter J, Richardt G, Hennersdorf M, Empen K, Fuernau G, Desch S, Eitel I, Hambrecht R, Fuhrmann J, Bohm M, Ebelt H, Schneider S, Schuler G, Werdan K: Intraaortic balloon support for myocardial infarction with cardiogenic shock. N Engl J Med 2012, 367:1287-1296.

4. Nowak-Machen M, Rawn JD, Shekar PS, Mitani A, Tuli S, Bingold TM, Lawlor G, Eltzschig HK, Shernan SK, Rosenberger P: Descending aortic calcification increases renal dysfunction and in-hospital mortality in cardiac surgery patients with intraaortic balloon pump counterpulsation placed perioperatively: a case control study. Crit Care 2012, 16:R17.

5. Belohlavek J, Mlcek M, Huptych M, Svoboda T, Havranek S, Ost'adal P, Boucek T, Kovarnik T, Mlejnsky F, Mrazek V, Belohlavek M, Aschermann M, Linhart A, Kittnar O: Coronary versus carotid blood flow and coronary perfusion pressure in a pig model of prolonged cardiac arrest treated by different modes of venoarterial ECMO and intraaortic balloon counterpulsation. Crit Care 2012, 16:R50.

6. Rimachi R, Bruzzi DC, Orellano-Jimenez C, Cotton F, Vincent J, De Backer D: Lactate/pyruvate ratio as a marker of tissue hypoxia in circulatory and septic shock. Anaesth Intensive Care 2012, 40:427-432.

7. Michaeli B, Martinez A, Revelly JP, Cayeux MC, Chiolero RL, Tappy L, Berger MM: Effects of endotoxin on lactate metabolism in humans. Crit Care 2012, 16:R139

8. Haji-Michael PG, Ladriere L, Sener A, Vincent JL, Malaisse WJ: Leukocyte glycolysis and lactate output in animal sepsis and ex vivo human blood. Metabolism 1999, 48:779-785.

9. Protti A, Russo R, Tagliabue P, Vecchio S, Singer M, Rudiger A, Foti G, Rossi A, Mistraletti G, Gattinoni L: Oxygen consumption is depressed in patients with lactic acidosis due to biguanide intoxication. Crit Care 2010, 14:R22.

10. Protti A, Fortunato F, Monti M, Vecchio S, Gatti S, Comi GP, De Giuseppe R, Gattinoni L: Metformin overdose, but not lactic acidosis per se, inhibits oxygen consumption in pigs. Crit Care 2012, 16:R75.

11. Protti A, Lecchi A, Fortunato F, Artoni A, Greppi N, Vecchio S, Fagiolari G, Moggio M, Comi GP, Mistraletti G, Lanticina B, Faraldi L, Gattinoni L: Metformin overdose causes platelet mitochondrial dysfunction in humans. Crit Care 2012, 16:R180.

12. Tsaknis G, Siempos II, Kopterides P, Maniatis NA, Magkou C, Kardara M, Panoutsou S, Kotanidou A, Roussos C, Armaganidis A: Metformin attenuates ventilator-induced lung injury. Crit Care 2012, 16:R134

13. Fazekas AS, Wewalka M, Zauner C, Funk GC: Carboxyhemoglobin levels in medical intensive care patients: a retrospective, observational study. Crit Care 2012, 16:R6.

14. Kiefer N, Hofer CK, Marx G, Geisen M, Giraud R, Siegenthaler N, Hoeft A, Bendjelid K, Rex S: Clinical validation of a new thermodilution system for the assessment of cardiac output and volumetric parameters. Crit Care 2012, 16:R98.

15. Chew MS, Ihrman L, During J, Bergenzaun L, Ersson A, Unden J, Ryden J, Akerman E, Larsson M: Extravascular lung water index improves the diagnostic accuracy of lung injury in patients with shock. Crit Care 2012, 16:R1.

16. Jozwiak M, Silva S, Persichini R, Anguel N, Osman D, Richard C, Teboul JL, Monnet $X$ : Extravascular lung water is an independent prognostic factor in patients with acute respiratory distress syndrome. Crit Care Med 2013, 42:472-480

17. Monnet X, Picard F, Lidzborski E, Mesnil M, Duranteau J, Richard C, Teboul $J$ : The estimation of cardiac output by the Nexfin device is of poor reliability for tracking the effects of a fluid challenge. Crit Care 2012, 16: R212.

18. Muller L, Bobbia X, Toumi M, Louart G, Molinari N, Ragonnet B, Quintard H, Leone M, Zoric L, Lefrant JY: Respiratory variations of inferior vena cava diameter to predict fluid responsiveness in spontaneously breathing patients with acute circulatory failure: need for a cautious use. Crit Care 2012, 16:R188

19. Bodson L, Vieillard-Baron A: Respiratory variation in inferior vena cava diameter: surrogate of central venous pressure or parameter of fluid responsiveness? Let the physiology reply. Crit Care 2012, 16:181.
20. Antonelli M, Levy M, Andrews PJ, Chastre J, Hudson LD, Manthous C, Meduri GU, Moreno RP, Putensen C, Stewart T, Torres A: Hemodynamic monitoring in shock and implications for management: International Consensus Conference, Paris, France, 27-28 April 2006. Intensive Care Med 2007, 33:575-590.

21. Vincent JL, Rhodes A, Perel A, Martin GS, Della RG, Vallet B, Pinsky MR, Hofer CK, Teboul JL, de Boode WP, Scolletta S, Vieillard-Baron A, De Backer D, Walley KR, Maggiorini M, Singer M: Clinical review: Update on hemodynamic monitoring - a consensus of 16. Crit Care 2011, 15:229.

22. Biais M, Carrie C, Delaunay F, Morel N, Revel P, Janvier G: Evaluation of a new pocket echoscopic device for focused-cardiac ultrasonography in emergency setting. Crit Care 2012, 16:R82.

23. Amiel JB, Grumann A, Lheritier G, Clavel M, Francois B, Pichon N, Dugard A, Marin B, Vignon P: Assessment of left ventricular ejection fraction using an ultrasonic stethoscope in critically ill patients. Crit Care 2012, 16:R29.

24. Bustam A, Noor AM, Singh VR, Arumugam K, Loch A: Performance of emergency physicians in point-of-care echocardiography following limited training. Emerg Med J 2013 [Epub ahead of print]

25. Weng L, Liu YT, Du B, Zhou JF, Guo XX, Peng JM, Hu XY, Zhang SY, Fang Q, Zhu WL: The prognostic value of left ventricular systolic function measured by tissue Doppler imaging in septic shock. Crit Care 2012, 16:R71.

26. Levy RJ, Piel DA, Acton PD, Zhou R, Ferrari VA, Karp JS, Deutschman CS: Evidence of myocardial hibernation in the septic heart. Crit Care Med 2005, 33:2752-2756

27. Lamia B, Maizel J, Ochagavia A, Chemla D, Osman D, Richard C, Teboul JL: Echocardiographic diagnosis of pulmonary artery occlusion pressure elevation during weaning from mechanical ventilation. Crit Care Med 2009, 37:1696-1701.

28. Caille V, Amiel JB, Charron C, Belliard G, Vieillard-Baron A, Vignon P: Echocardiography: a help in the weaning process. Crit Care 2010, 14:R120.

29. Porhomayon J, Papadakos P, Nader ND: Failed weaning from mechanical ventilation and cardiac dysfunction. Crit Care Res Pract 2012, 2012:173527.

30. Moschietto S, Doyen D, Grech L, Dellamonica J, Hyvernat H, Bernardin G: Transthoracic echocardiography with Doppler tissue imaging predicts weaning failure from mechanical ventilation: evolution of the left ventricle relaxation rate during a spontaneous breathing trial is the key factor in weaning outcome. Crit Care 2012, 16:R81.

31. Testa A, Soldati G, Copetti R, Giannuzzi R, Portale G, Gentiloni-Silveri N: Early recognition of the 2009 pandemic influenza A (H1N1) pneumonia by chest ultrasound. Crit Care 2012, 16:R30.

32. Tsung JW, Kessler DO, Shah VP: Prospective application of clinicianperformed lung ultrasonography during the 2009 H1N1 influenza A pandemic: distinguishing viral from bacterial pneumonia. Crit Ultrasound J 2012, 4:16

33. Yeh YC, Wang MJ, Lin CP, Fan SZ, Tsai JC, Sun WZ, Ko WJ: Enoxaparin sodium prevents intestinal microcirculatory dysfunction in endotoxemic rats. Crit Care 2012, 16:R59.

34. Iba T, Okamoto K, Ohike T, Tajirika T, Aihara K, Watanabe S, Kayhanian H: Enoxaparin and fondaparinux attenuates endothelial damage in endotoxemic rats. J Trauma Acute Care Surg 2012, 72:177-182.

35. Wexler O, Morgan MA, Gough MS, Steinmetz SD, Mack CM, Darling DC, Doolin KP, Apostolakos MJ, Graves BT, Frampton MW, Chen X, Pietropaol AP: Brachial artery reactivity in patients with severe sepsis: an observational study. Crit Care 2012, 16:R38.

36. Haase M, Haase-Fielitz A, Bellomo R, Devarajan P, Story D, Matalanis G, Reade MC, Bagshaw SM, Seevanayagam N, Seevanayagam S, Doolan L, Buxton B, Dragun D: Sodium bicarbonate to prevent increases in serum creatinine after cardiac surgery: a pilot double-blind, randomized controlled trial. Crit Care Med 2009, 37:39-47.

37. Heringlake M, Heinze H, Schubert M, Novak Y, Guder J, Kleinebrahm M, Paarmann $H$, Hanke T, Schon J: A perioperative infusion of sodium bicarbonate does not improve renal function in cardiac surgery patients: a prospective observational cohort study. Crit Care 2012, 16:R156

38. Kristeller JL, Zavorsky GS, Prior JE, Keating DA, Brady MA, Romaldini TA, Hickman TL, Stahl RF: Lack of effectiveness of sodium bicarbonate in preventing kidney injury in patients undergoing cardiac surgery: a randomized controlled trial. Pharmacotherapy 2013, 33:710-717. 
39. Haase M, Haase-Fielitz A, Plass M, Kuppe H, Hetzer R, Hannon C, Murray PT, Bailey MJ, Bellomo R, Bagshaw SM: Prophylactic perioperative sodium bicarbonate to prevent acute kidney injury following open heart surgery: a multicenter double-blinded randomized controlled trial. PLoS Med 2013, 10:e1001426.

40. Meijers B, Laleman W, Vermeersch P, Nevens F, Wilmer A, Evenepoel P: A prospective randomized open-label crossover trial of regional citrate anticoagulation vs. anticoagulation free liver dialysis by the Molecular Adsorbents Recirculating System. Crit Care 2012, 16:R20.

$10.1186 / \mathrm{cc} 13127$

Cite this article as: De Backer: Year in review 2012: Critical Care - cardiology. Critical Care 2013, 17:247 